Supporting Information

\title{
Cooccurrence of and Infant Exposure to Multiple Common and Unusual Phenolic Antioxidants in Human Breast Milk
}

Yun Zhang, Bibai Du, Jiali Ge, Liangying Liu, Mingshan Zhu, Juan Li, and Lixi Zeng*

Guangdong Key Laboratory of Environmental Pollution and Health, School of Environment, Jinan University, Guangzhou 511443, China

*Corresponding author

Dr. Lixi Zeng

Guangdong Key Laboratory of Environmental Pollution and Health

School of Environment, Jinan University

Guangzhou 511443, China

Tel: +(86)-20-37336629

Fax: +(86)-20-37336629

E-mail: $\underline{1 x z e n g @ j \text { jnu.edu.cn }}$ 


\section{Materials}

Eight commonly used SPAs, that are BHT, DBP, BHA, AO 246, DTBSBP, D $t$ AP, 4- $t$ OP, and AO 2246, were purchased from TCI (Japan). Two high molecular weight SPAs, Irganox 1076 and Irganox 1135, were obtained from TCI (Japan) and Toronto Research Chemicals (Canada). Four major TPs of BHT, namely, BHT-OH, BHT-CHO, BHT-Q, and BHT-quinol, were purchased from Sigma-Aldrich (USA). Two isotope-labeled standards, 2,6-di-(tertbutyl-d9)-4-methyl(phenol-3,5,O-d3) $\left(\mathrm{BHT}-\mathrm{d}_{21}\right)$ and ${ }^{13} \mathrm{C}_{12}$-butyl paraben $\left({ }^{13} \mathrm{C}_{12}\right.$-BuP) were purchased from Cambridge Isotope Laboratories (USA) and Toronto Research Chemicals (Canada), respectively. HPLC-grade methanol was supplied by Merck (Darmstadt, Germany). HPLC-grade acetonitrile (ACN) and $n$-hexane (Hex) were acquired from Oceanpak (Sweden). Ultra-pure water was prepared using a Milli-Q ${ }^{\circledR}$ Integral system (Millipore, USA).

\section{Sample Preparation and Quantitative Analysis}

Human breast milk samples were allowed to thaw at room temperature in the dark. The lipid, protein, and lactose contents were measured using a MIRA infrared (IR) milk analyzer (Bruker, Karlsruhe, German). For the analysis of SPAs, $1.5 \mathrm{~g}$ of accurately weighted breast milk sample (spiked with $10 \mathrm{ng}$ of BHT- $\mathrm{d}_{21}$ and $1 \mathrm{ng}$ of ${ }^{13} \mathrm{C}_{12}-\mathrm{BuP}$ ) was transferred into a clean glass tube. After vortex-mixing and then incubating at $4{ }^{\circ} \mathrm{C}$ overnight, $8 \mathrm{~mL}$ of a mixed solvent of acetonitrile and $n$-hexane (3:5 in volume) were added into the glass tube and subjected to shake at $300 \mathrm{rpm}$ for $10 \mathrm{~min}$. Subsequently, the mixture was ultrasonically extracted for $20 \mathrm{~min}$. The supernatant organic layer was transferred into another clean glass tube after centrifugation at $4500 \mathrm{rpm}$ for $10 \mathrm{~min}$. The extraction process was repeated four times, and the combined extracts were condensed to $1 \mathrm{~mL}$ under a gentle nitrogen stream. A Florisil cartridge (Supelco, $500 \mathrm{mg} / 3.0 \mathrm{~mL}$ ) was adopted for further purification. The cartridge was preconditioned with $9 \mathrm{~mL}$ of $n$-hexane. Upon loading of the extract, the target compounds were eluted with $12 \mathrm{~mL}$ of $n$-hexane. The elute was concentrated to $0.5 \mathrm{~mL}$ under a gentle nitrogen stream, and then separated into $0.1 \mathrm{~mL}$ and $0.4 \mathrm{~mL}$ aliquots. The $0.1 \mathrm{ml}$ sample (in $n$-hexane) was analyzed for BHT, AO 246, and DTBSBP by gas chromatography-mass spectrometry (GC-MS). The $0.4 \mathrm{~mL}$ sample was solvent-exchanged to methanol, filtered through a $0.22 \mu \mathrm{m}$ PTFE filter membrane, and then analyzed for DBP, 
BHA, DtAP, 4-tOP, AO 2246, Irganox 1076, Irganox 1135, BHT-OH, BHT-CHO, BHT-Q, and BHT-quinol by liquid chromatography-tandem mass spectrometry (LC-MS/MS).

In the present study, eight common SPAs and four TPs were quantitatively analyzed based on previously reported methods, ${ }^{1-6}$ with some optimizations. The quantitative analysis for two unusual SPAs was developed based on LC-MS/MS with electrospray ionization (ESI) in negative-ion multiple reaction monitoring (MRM) mode. BHT, AO 246, and DTBSBP were analyzed using a 7890B gas chromatograph coupled with a 5977B electron impact mass spectrometer (Agilent, USA) in selected ion monitoring (SIM) mode. An HP-5MS fused-silica capillary column $(30 \mathrm{~m} \times 0.25 \mathrm{~mm}$ i.d. $\times 0.25 \mathrm{~mm}$ film thickness $)$ was selected for chromatographic separation. The remaining five common SPAs, four TPs, and two unusual SPAs were analyzed using an ExionLC AC ultrafast liquid chromatograph interfaced with a Triple Quad ${ }^{\mathrm{TM}} 5500$ mass spectrometer (AB Sciex, USA) with ESI in negative-ion MRM mode. An XBridge ${ }^{\circledR}$ BEH C18 column $(2.5 \mu \mathrm{m}, 2.1 \mathrm{~mm} \times 100 \mathrm{~mm}$, Waters $)$ connected to an XBridge ${ }^{\circledR}$ BEH C18 $\operatorname{VanGuard~}{ }^{\circledR}$ cartridge $(2.5 \mu \mathrm{m}, 2.1 \mathrm{~mm} \times 5 \mathrm{~mm}$, Waters $)$ was used for chromatographic separation. Detailed information on the optimized MS parameters and the qualitative/quantitative monitored ions or MRM ion pairs are given in Table S2. Representative GC and LC chromatograms of the target analytes are shown in Figure S2 and $\mathrm{S} 3$, respectively. 
Table S1. Name, CAS No, formula, and key physicochemical properties of 14 target chemicals.

\begin{tabular}{|c|c|c|c|c|c|c|c|c|c|}
\hline Full Name & Abbreviation & CAS & Formula & MW & $\log K_{\text {ow }}^{a}$ & $\mathrm{p} K \mathrm{a}^{b}$ & $\begin{array}{l}\text { Rapid Biodegradation } \\
\text { Probability (Biowin1) }\end{array}$ & $\begin{array}{l}\text { Biotransformation } \\
\text { Half-life (days) }{ }^{a}\end{array}$ & $\mathrm{BCF}^{c}$ \\
\hline 2,6-di-tert-butyl-4-methylphenol & BHT & $128-37-0$ & $\mathrm{C}_{15} \mathrm{H}_{24} \mathrm{O}$ & 220.18 & 5.03 & 12.76 & 0.4453 & 1.154 & 4990 \\
\hline 2,4-di-tert-butylphenol & DBP & $96-76-4$ & $\mathrm{C}_{14} \mathrm{H}_{22} \mathrm{O}$ & 206.17 & 5.33 & 11.56 & 0.3973 & 2.002 & 1870 \\
\hline 2,4,6-tri-tert-butylphenol & AO 246 & $732-26-3$ & $\mathrm{C}_{18} \mathrm{H}_{30} \mathrm{O}$ & 262.23 & 6.39 & 12.61 & 0.1866 & 3.939 & 6310 \\
\hline 2,6-di-tert-butyl-4-sec-butylphenol & DTBSBP & $17540-75-9$ & $\mathrm{C}_{18} \mathrm{H}_{30} \mathrm{O}$ & 262.23 & 6.43 & 11.85 & 0.4252 & 3.173 & 7500 \\
\hline 3-tert-butyl-4-hydroxyanisole & BHA & $121-00-6$ & $\mathrm{C}_{11} \mathrm{H}_{16} \mathrm{O}_{2}$ & 180.12 & 3.50 & 11.83 & 0.7255 & 0.3878 & 131 \\
\hline 2,4-di-tert-amylphenol & $\mathrm{D} t \mathrm{AP}$ & $120-95-6$ & $\mathrm{C}_{16} \mathrm{H}_{26} \mathrm{O}$ & 234.20 & 6.31 & 11.00 & 0.3839 & 4.2 & 11200 \\
\hline 4-tert-octylphenol & $4-t \mathrm{OP}$ & $140-66-9$ & $\mathrm{C}_{14} \mathrm{H}_{22} \mathrm{O}$ & 206.17 & 5.28 & 10.15 & 0.3973 & 2.361 & 5090 \\
\hline $\begin{array}{l}\text { 2,2'-Methylenebis(6-tert-butyl-4-methylph } \\
\text { enol) }\end{array}$ & AO 2246 & $119-47-1$ & $\mathrm{C}_{23} \mathrm{H}_{32} \mathrm{O}_{2}$ & 340.24 & 7.97 & 11.32 & 0.6132 & 0.4703 & 249,000 \\
\hline $\begin{array}{l}\text { Octadecyl-3,5-di-tert-butyl-4-hydroxy-ben } \\
\text { zenepropanoate }\end{array}$ & Irganox 1076 & $2082-79-3$ & $\mathrm{C}_{35} \mathrm{H}_{62} \mathrm{O}_{3}$ & 530.47 & 13.41 & 12.33 & 0.5800 & 33.46 & $1,000,000$ \\
\hline $\begin{array}{l}\text { Octyl-3,5-di-tert-butyl-4-hydroxyhydrocin } \\
\text { namate }\end{array}$ & Irganox 1135 & $125643-61-0$ & $\mathrm{C}_{25} \mathrm{H}_{42} \mathrm{O}_{3}$ & 390.31 & 7.93 & - & 0.5451 & 0.8763 & - \\
\hline 2,6-di-tert-butyl-4-(hydroxymethyl)phenol & BHT-OH & $88-26-6$ & $\mathrm{C}_{15} \mathrm{H}_{24} \mathrm{O}_{2}$ & 236.18 & 3.56 & 12.01 & 0.5417 & 0.1147 & 271 \\
\hline 3,5-di-tert-butyl-4-hydroxybenzaldehyde & BHT-CHO & $1620-98-0$ & $\mathrm{C}_{15} \mathrm{H}_{22} \mathrm{O}_{2}$ & 234.16 & 4.20 & 8.33 & 0.6686 & 0.8037 & 835 \\
\hline 2,6-di-tert-butyl-p-benzoquinone & BHT-Q & $719-22-2$ & $\mathrm{C}_{14} \mathrm{H}_{20} \mathrm{O}_{2}$ & 220.15 & 4.42 & - & 0.2885 & 0.9285 & 434 \\
\hline $\begin{array}{l}\text { 2,6-di-tert-butyl-4-hydroxy-4-methyl-2,5- } \\
\text { cyclohexadienone }\end{array}$ & BHT-quinol & $10396-80-2$ & $\mathrm{C}_{15} \mathrm{H}_{24} \mathrm{O}_{2}$ & 236.18 & 3.72 & 12.29 & - & - & 54.3 \\
\hline
\end{tabular}

${ }^{a}$ Predicted physicochemical properties by US EPA EPI Suite (V4.11). ${ }^{b}$ Calculated using Advanced Chemistry Development (ACD/Labs) Software V11.02 (C 1994-2020 ACD/Labs).

${ }^{c}$ BCF: bioconcentration factor; calculated using Advanced Chemistry Development (ACD/Labs) Software V11.02 (C 1994-2020 ACD/Labs). 
Table S2. Optimized MS Parameters for the qualitative and quantitative analysis.

\begin{tabular}{|c|c|c|c|c|c|}
\hline $\begin{array}{c}\text { Instrument } \\
\text { method }\end{array}$ & Compound & $\begin{array}{l}\text { Precursor ion } \\
(\mathbf{m} / \mathbf{z})\end{array}$ & $\begin{array}{l}\text { Product ion } \\
\qquad(\mathbf{m} / \mathbf{z})\end{array}$ & $\begin{array}{c}\text { Declustering } \\
\text { potential (V) }\end{array}$ & $\begin{array}{c}\text { Collision } \\
\text { energy (V) }\end{array}$ \\
\hline \multirow{23}{*}{ LC-MS/MS } & \multirow{2}{*}{ BHA } & \multirow{2}{*}{179.0} & $164.1^{*}$ & -40 & -20 \\
\hline & & & 149.1 & -40 & -34 \\
\hline & \multirow{2}{*}{ DBP } & \multirow{2}{*}{205.1} & $189.1^{*}$ & -100 & -35 \\
\hline & & & 173.1 & -100 & -54 \\
\hline & \multirow{2}{*}{$\mathrm{D} t \mathrm{AP}$} & \multirow{2}{*}{233.1} & $203.1^{*}$ & -130 & -42 \\
\hline & & & 217.2 & -130 & -34 \\
\hline & \multirow{2}{*}{$4-t \mathrm{OP}$} & \multirow{2}{*}{205.2} & $133.1^{*}$ & -90 & -30 \\
\hline & & & 93.0 & -90 & -62 \\
\hline & \multirow{2}{*}{ AO 2246} & \multirow{2}{*}{338.7} & $162.9^{*}$ & -70 & -38 \\
\hline & & & 183.0 & -70 & -30 \\
\hline & \multirow{2}{*}{ ВНT-OH } & \multirow{2}{*}{234.9} & $217.2 *$ & -55 & -27 \\
\hline & & & 160.1 & -48 & -35 \\
\hline & \multirow{2}{*}{ BHT-CHO } & \multirow{2}{*}{233.2} & $217.2^{*}$ & -90 & -45 \\
\hline & & & 203.2 & -90 & -43 \\
\hline & \multirow{2}{*}{ BHT-Q } & \multirow{2}{*}{220.1} & $205.1 *$ & -100 & -33 \\
\hline & & & 148.0 & -100 & -41 \\
\hline & \multirow{2}{*}{ BHT-quinol } & \multirow{2}{*}{235.1} & $220.2 *$ & -90 & -24 \\
\hline & & & 205.1 & -90 & -40 \\
\hline & \multirow{2}{*}{ Irganox 1076} & \multirow{2}{*}{529.5} & $267.2 *$ & -80 & -57 \\
\hline & & & 311.3 & -80 & -36 \\
\hline & \multirow{2}{*}{ Irganox 1135} & \multirow{2}{*}{389.3} & $171.1^{*}$ & -90 & -30 \\
\hline & & & 40.9 & -90 & -76 \\
\hline & ${ }^{13} \mathrm{C}_{12}-\mathrm{BuP}$ & 199.0 & 98.0 & -50 & -30 \\
\hline \multirow{4}{*}{ GC-MS } & BHT & \multicolumn{2}{|c|}{$220.2 / 205.2 / 177.2$} & - & - \\
\hline & AO 246 & \multicolumn{2}{|c|}{$262.3 / 247.2$} & - & - \\
\hline & DTBSBP & \multicolumn{2}{|c|}{$262.3 / 247.2 / 233.3$} & - & - \\
\hline & BHT- $\mathrm{d}_{21}$ & \multicolumn{2}{|c|}{$240.3 / 222.3$} & - & - \\
\hline
\end{tabular}

* quantitative ion 
Table S3. QA/QC data, including matrix spike recoveries, matrix effect (ME), MQL, and procedure blank, for the target analytes in breast milk samples.

\begin{tabular}{|c|c|c|c|c|c|c|c|c|}
\hline \multirow{2}{*}{ Compound } & \multicolumn{2}{|c|}{$\begin{array}{l}\text { Spiked level } \\
\text { (1 ng/mL) }\end{array}$} & \multicolumn{2}{|c|}{$\begin{array}{l}\text { Spiked level } \\
(5 \mathrm{ng} / \mathrm{mL})\end{array}$} & \multirow{2}{*}{$\begin{array}{l}\text { ME } \\
(\%)\end{array}$} & \multirow{2}{*}{$\begin{array}{c}\text { RSD } \\
(\%, n=3)\end{array}$} & \multirow{2}{*}{$\begin{array}{l}\text { MQL } \\
\text { (ng/g) }\end{array}$} & \multirow{2}{*}{$\begin{array}{c}\text { Blank } \\
(\mathrm{ng} / \mathrm{mL})\end{array}$} \\
\hline & $\begin{array}{c}\text { Recovery } \\
(\%)\end{array}$ & $\begin{array}{c}\text { RSD } \\
(\%, n=3)\end{array}$ & $\begin{array}{c}\text { Recovery } \\
(\%)\end{array}$ & $\begin{array}{c}\text { RSD } \\
(\%, n=3)\end{array}$ & & & & \\
\hline BHT & 86 & 3 & 85 & 4 & 98 & 3 & 0.13 & 0.14 \\
\hline AO 246 & 82 & 6 & 83 & 5 & 99 & 4 & 0.061 & nd \\
\hline DTBSBP & 89 & 3 & 87 & 4 & 92 & 4 & 0.021 & nd \\
\hline BHA & 76 & 11 & 78 & 10 & 101 & 3 & 0.023 & nd \\
\hline DBP & 86 & 11 & 89 & 9 & 94 & 10 & 1.20 & 1.98 \\
\hline $\mathrm{D} t \mathrm{AP}$ & 80 & 7 & 82 & 6 & 102 & 2 & 0.031 & nd \\
\hline $4-t \mathrm{OP}$ & 90 & 8 & 90 & 9 & 97 & 9 & 0.043 & nd \\
\hline AO 2246 & 95 & 6 & 93 & 7 & 85 & 6 & 0.006 & nd \\
\hline Irganox 1076 & 58 & 9 & 62 & 8 & 81 & 12 & 0.79 & 1.05 \\
\hline Irganox 1135 & 72 & 3 & 74 & 5 & 95 & 7 & 0.008 & nd \\
\hline BHT-OH & 72 & 8 & 73 & 7 & 98 & 9 & 0.018 & nd \\
\hline BHT-CHO & 86 & 4 & 85 & 5 & 93 & 6 & 0.070 & nd \\
\hline BHT-Q & 88 & 6 & 90 & 6 & 93 & 4 & 0.097 & nd \\
\hline BHT-quinol & 84 & 7 & 85 & 6 & 98 & 9 & 0.093 & nd \\
\hline${ }^{13} \mathrm{C}_{12}-\mathrm{BuP}$ & 80 & 4 & 81 & 5 & 98 & 8 & - & nd \\
\hline BHT- $\mathrm{d}_{21}$ & 89 & 5 & 90 & 5 & 96 & 2 & - & nd \\
\hline
\end{tabular}

nd: not detected 
Table S4. Spearman's correlation matrix for SPA and TP concentrations and lipid content.

\begin{tabular}{|c|c|c|c|c|c|c|c|}
\hline & $\begin{array}{c}\text { Lipid } \\
\text { content }(\%)\end{array}$ & DBP & BHT & BHT-OH & BHT-CHO & BHT-Q & BHT-quinol \\
\hline $\begin{array}{c}\text { Lipid } \\
\text { content }(\%)\end{array}$ & 1 & & & & & & \\
\hline DBP & $0.272^{*}$ & 1 & & & & & \\
\hline BHT & $0.251^{*}$ & $0.297^{* *}$ & 1 & & & & \\
\hline ВНT-OH & 0.042 & $0.380^{* *}$ & $0.428^{* *}$ & 1 & & & \\
\hline BHT-CHO & 0.122 & $0.668^{* *}$ & $0.414^{* *}$ & $0.546^{* *}$ & 1 & & \\
\hline BHT-Q & 0.133 & $0.423^{* *}$ & $0.303^{* *}$ & $0.434^{* *}$ & $0.544^{* *}$ & 1 & \\
\hline $\begin{array}{c}\text { BHT-quino } \\
1\end{array}$ & $0.236^{*}$ & $0.313^{* *}$ & $0.261^{*}$ & $0.445^{* *}$ & $0.398^{* *}$ & $0.668^{* *}$ & 1 \\
\hline
\end{tabular}

** Correlation is at significant level of $p<0.01$ (two-tailed).

${ }^{*}$ Correlation is at significant level of $p<0.05$ level (two-tailed). 
Table S5. Multivariable linear regression coefficients [adjusted $\beta,(95 \%$ confidence intervals)] between $\log _{10}$-transformed SPA concentrations in breast milk and mother's eating habit, PCP use, and education level. The models adjusted for maternal age, parity, and passive smoking status.

\begin{tabular}{|c|c|c|c|}
\hline & BHT & DBP & $\sum \mathrm{TPs}$ \\
\hline \multicolumn{4}{|l|}{ Eating habit } \\
\hline mainly fresh foods & reference & reference & reference \\
\hline mainly prepackaged foods & $0.522(0.364,0.681)^{a}$ & $0.716(0.429,1.003)$ & $0.416(0.257,0.575)$ \\
\hline both of them & $0.182(0.054,0.311)$ & $0.444(0.208,0.679)$ & $0.161(0.030,0.292)$ \\
\hline$p$ for trend & $<0.05$ & $<0.001$ & $<0.05$ \\
\hline \multicolumn{4}{|l|}{ Use status of PCPs } \\
\hline$<6$ PCPs used daily & reference & reference & reference \\
\hline$\geq 6$ PCPs used daily & $0.195(0.061,0.329)$ & $0.235(0.028,0.442)$ & $0.154(0.028,0.280)$ \\
\hline$p$ for trend & $<0.05$ & $<0.05$ & $<0.05$ \\
\hline \multicolumn{4}{|l|}{ Maternal education } \\
\hline less than high school & reference & reference & reference \\
\hline high school & $0.130(-0.105,0.364)$ & $0.086(-0.341,0.514)$ & $0.261(0.040,0.482)$ \\
\hline college or higher & $0.245(0.024,0.466)$ & $0.462(0.064,0.861)$ & $0.278(0.071,0.486)$ \\
\hline$p$ for trend & $<0.05$ & $<0.05$ & $<0.05$ \\
\hline
\end{tabular}

${ }^{a}$ The bold indicates the significance at $p<0.05$ or $p<0.001$. 
Table S6. Binary logistical regression coefficients [adjusted OR, (95\% confidence intervals)] between newborn birth outcomes (prematurity, birth weight, and birth length) and SPA concentrations (ng/mL) in breast milk. Adjusted covariates: maternal age, parity, passive smoking status, and newborn gender for prematurity ( $<37$ or $\geq 37$ weeks); maternal age, parity, passive smoking status, newborn gender, and prematurity status for birth weight ( $<3$ or $\geq 3 \mathrm{~kg}$ ) and birth length $(<50$ or $\geq 50 \mathrm{~cm})$.

\begin{tabular}{|c|c|c|c|}
\hline & $\begin{array}{l}\text { Prematurity } \\
(<37 \text { weeks })\end{array}$ & $\begin{array}{l}\text { Birth weight } \\
\quad(<3 \mathrm{~kg})\end{array}$ & $\begin{array}{l}\text { Birth length } \\
(<50 \mathrm{~cm})\end{array}$ \\
\hline \multicolumn{4}{|l|}{ BHT } \\
\hline$<0.87$ & reference & reference & reference \\
\hline $0.87-1.54$ & $3.360(0.591,19.083)$ & $1.560(0.295,8.253)$ & $1.366(0.288,6.487)$ \\
\hline$>1.54$ & $6.028(1.146,31.714)^{a}$ & $1.667(0.314,8.852)$ & $5.417(1.187,24.728)$ \\
\hline$p$ for trend & $<0.05$ & $>0.05$ & $<0.05$ \\
\hline \multicolumn{4}{|l|}{ DBP } \\
\hline$<10.3$ & reference & reference & reference \\
\hline $10.3-21.4$ & $0.420(0.104,1.697)$ & $0.701(0.145,3.378)$ & $3.319(0.592,18.623)$ \\
\hline$>21.4$ & $0.215(0.045,1.035)$ & $0.938(0.195,4.510)$ & $3.359(0.607,18.599)$ \\
\hline$p$ for trend & $>0.05$ & $>0.05$ & $>0.05$ \\
\hline \multicolumn{4}{|l|}{$\sum \mathrm{TPs}$} \\
\hline$<1.53$ & reference & reference & reference \\
\hline $1.53-2.61$ & $1.462(0.335,6.382)$ & $1.732(0.351,8.543)$ & $1.209(0.231,6.330)$ \\
\hline$>2.61$ & $1.783(0.451,7.050)$ & $2.397(0.488,11.774)$ & $7.465(1.449,38.468)$ \\
\hline$p$ for trend & $>0.05$ & $>0.05$ & $<0.05$ \\
\hline
\end{tabular}

${ }^{a}$ The bold indicates the significance at $p<0.05$. 


\section{Synthetic Phenolic Antioxidants (SPAs)}

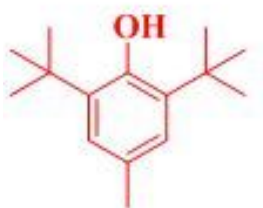

BHT<smiles>CC(C)(C)c1cc(C(C)(C)C)c(O)c(C(C)(C)C)c1</smiles>

AO 246<smiles>CCC(C)c1cc(C(C)(C)C)c(O)c(C(C)(C)C)c1</smiles>

DTBSBP<smiles>COc1ccc(O)c(C(C)(C)C)c1</smiles>

BHA<smiles>CC(C)(C)c1ccc(O)c(C(C)(C)C)c1</smiles>

DBP<smiles>CCC(C)(C)c1ccc(O)c(C(C)(C)CC)c1</smiles>

DtAP

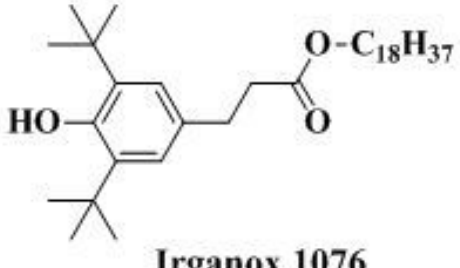

Irganox 1076<smiles>CC(C)(C)CC(C)(C)c1ccc(O)cc1</smiles><smiles>Cc1cc(Cc2cc(C)cc(C(C)(C)C)c2O)c(O)c(C(C)(C)C)c1</smiles>

4-tOP

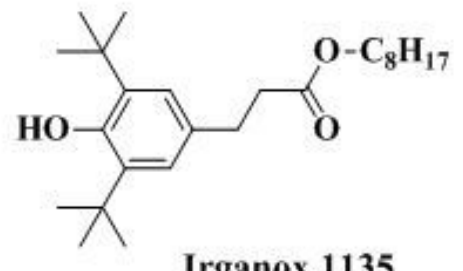

Irganox 1135

\section{Transformation Products (TPs)}<smiles>CC(C)(C)c1cc(CO)cc(C(C)(C)C)c1O</smiles>

BHT-OH<smiles>CC(C)(C)c1cc(C=O)cc(C(C)(C)C)c1O</smiles>

BHT-CHO<smiles>CC(C)(C)C1=CC(=O)C=C(C(C)(C)C)C1=O</smiles>

BHT-Q<smiles>CC1(O)C=C(C(C)(C)C)C(=O)C(C(C)(C)C)=C1</smiles>

BHT-quinol

Figure S1. Chemical structures and abbreviations of eight common and two unusual SPAs and four TPs. Molecular structures in red represent two the most abundant SPAs detected in human breast milk. 


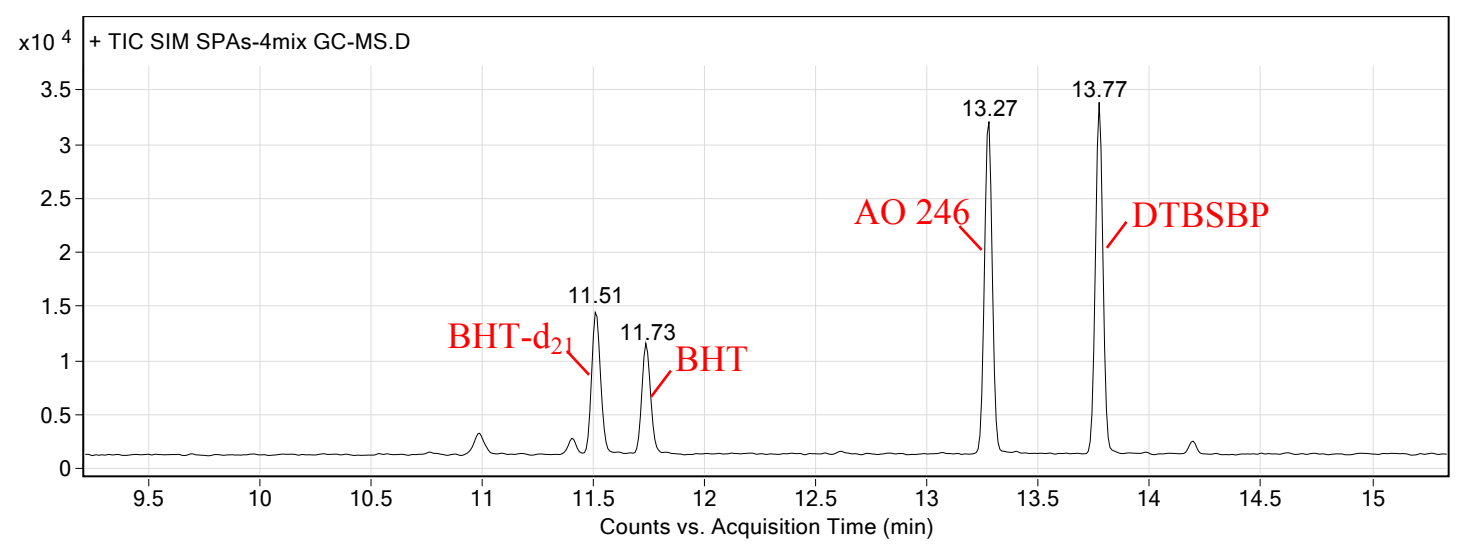

Figure S2. Representative GC-MS chromatogram of three target compounds. 


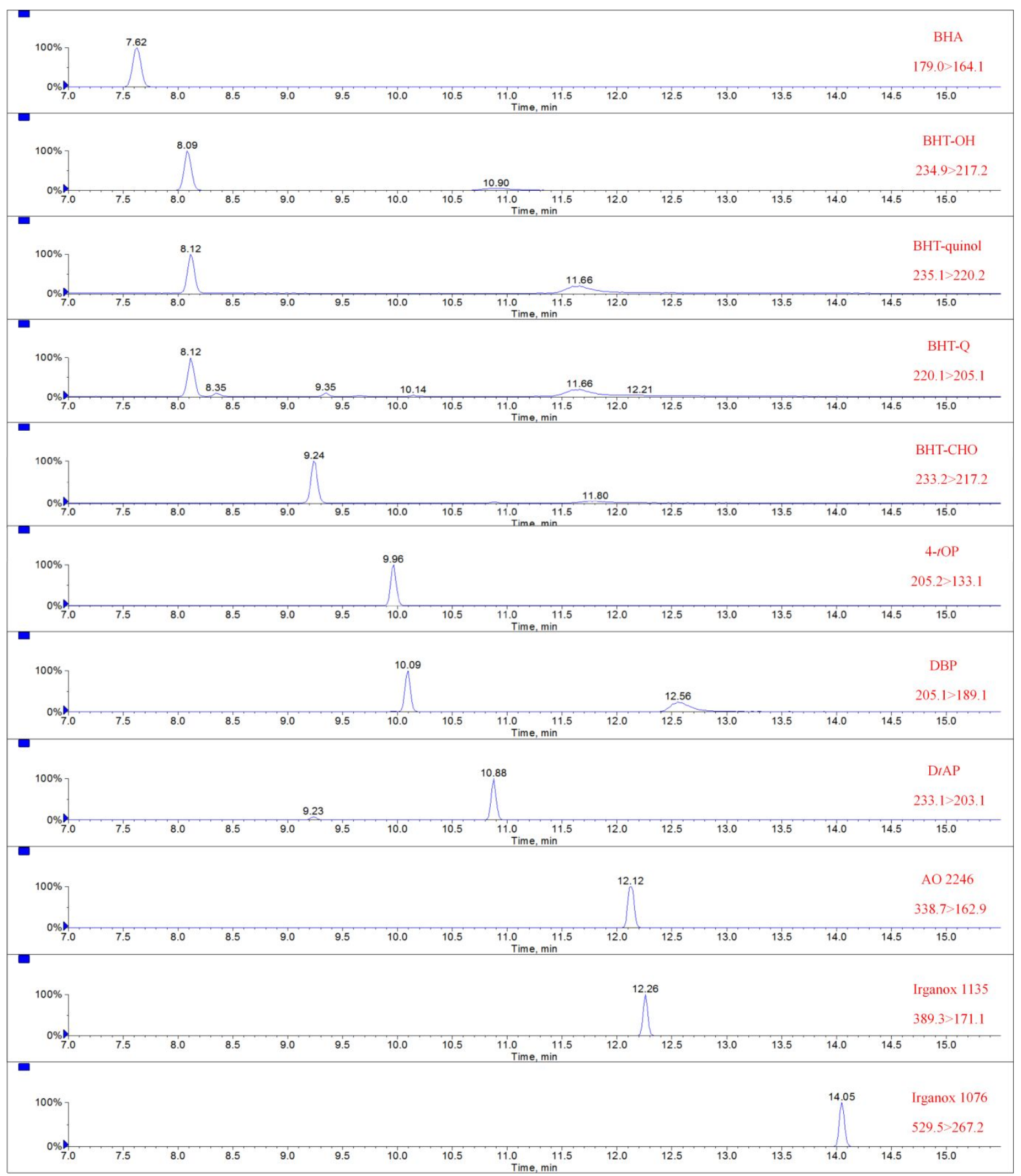

Figure S3. Representative LC-MS/MS chromatograms of eleven target compounds. 


\section{REFERENCES}

1. Du, B. B.; Zhang, Y.; Lam, J. C. W.; Pan, S. L.; Huang, Y. X.; Chen, B. W.; Lan, S. Y.; Li, J.; Luo, D.; Zeng, L. X., Prevalence, Biotransformation, and Maternal Transfer of Synthetic Phenolic Antioxidants in Pregnant Women from South China. Environ. Sci. Technol. 2019, doi: 10.1021/acs.est.9b04709.

2. Wang, W.; Asimakopoulos, A. G.; Abualnaja, K. O.; Covaci, A.; Gevao, B.; Johnson-Restrepo, B.; Kumosani, T. A.; Malarvannan, G.; Tu Binh, M.; Moon, H.-B.; Nakata, H.; Sinha, R. K.; Kannan, K., Synthetic Phenolic Antioxidants and Their Metabolites in Indoor Dust from Homes and Microenvironments. Environ. Sci. Technol. 2016, 50, (1), 428-434.

3. Liu, R.; Mabury, S. A., Synthetic Phenolic Antioxidants and Transformation Products in Human Sera from United States Donors. Environ. Sci. Technol. Lett. 2018, 5, (7), 419-423.

4. Wang, X.; Hou, X.; Zhou, Q.; Liao, C.; Jiang, G., Synthetic Phenolic Antioxidants and Their Metabolites in Sediments from the Coastal Area of Northern China: Spatial and Vertical Distributions. Environ. Sci. Technol. 2018, 52, (23), 13690-13697.

5. Wang, X.; Hou, X.; Hu, Y.; Zhou, Q.; Liao, C.; Jiang, G., Synthetic Phenolic Antioxidants and Their Metabolites in Mollusks from the Chinese Bohai Sea: Occurrence, Temporal Trend, and Human Exposure. Environ. Sci. Technol. 2018, 52, (17), 10124-10133.

6. Wang, W.; Kannan, K., Quantitative identification of and exposure to synthetic phenolic antioxidants, including butylated hydroxytoluene, in urine. Environ. Int. 2019, 128, 24-29. 